ENSAYOS

\title{
La escuela como espacio para el reconocimiento del patrimonio cultural de niños y niñas que han migrado*
}

\author{
The school as a space for the recognition of the cultural heritage \\ of children who have migrated
}

\author{
Susan Sanhueza Henríquez, Carmen Carmona Rodríguez, \\ Karla Rosalía Morales Mendozac \\ ${ }^{a}$ Facultad de Ciencias Sociales, Universidad de Chile. \\ susan.sanhueza@uchile.cl \\ ${ }^{b}$ Facultad de Educación, Universidad de Valencia, España. \\ carmen.carmona@uv.es \\ ${ }^{c}$ Universidad Católica del Maule, Chile. \\ kmorales@ucm.cl
}

\begin{abstract}
RESUMEN
A los niños y niñas cuyas familias han migrado se les introduce en las formas culturales dominantes, sin darles la oportunidad de aportar las suyas, ignorando la contribución que podrían hacer en la promoción de escuelas más inclusivas. Desde esta perspectiva, el trabajo tiene como objetivos: (1) analizar el concepto de patrimonio cultural problematizando sobre las distintas racionalidades que subyacen a su comprensión en el ámbito educativo; (2) aportar elementos de reflexión sobre identidad y reconocimiento a partir de una educación en patrimonio cultural y (3) proponer líneas de actuación para el trabajo en patrimonio cultural en las escuelas. Para dar respuesta a estos objetivos, hemos realizado una revisión documental desde la literatura especializada. Los resultados relevan el rol que tienen los "portadores de cultura" en la identificación de patrimonio cultural de niños y niñas migrantes, la necesidad de formar al profesorado en competencias que le permitan interpretar contextos de aprendizaje interculturales y la necesidad de crear puentes entre lo propio y lo ajeno, entre lo común y lo diferente para favorecer prácticas más inclusivas.
\end{abstract}

Palabras claves: patrimonio, identidad, reconocimiento, inclusión, interculturalidad, migración.

\begin{abstract}
Children whose families have migrated are introduced into dominant cultural forms, without giving them the opportunity to contribute their own, ignoring the contribution they could make in promoting schools that are more inclusive. From this perspective, the work aims to: (1) analyze the concept of cultural heritage by problematizing the different rationales that underlie its understanding in the educational field; (2) provide elements of reflection on identity and recognition from education in cultural heritage and (3) propose lines of action for work on cultural heritage in schools. To meet these objectives, we conducted a literature review of specialized literature. The results highlighted the role of "cultural bearers" in identifying the cultural heritage of migrant children, the need to train teachers in skills that allow them to interpret intercultural learning contexts and the need to create bridges between themselves and others, and between the common and the different to favor practices that are more inclusive.
\end{abstract}

Key words: heritage, identity, recognition, inclusion, interculturality, migration.

\footnotetext{
* Agradecimientos al proyecto FONDECYT regular $\mathrm{N}^{\circ} 1191045$ denominado "Respuesta educativa de la escuela ante la inmersión lingüística de estudiantes inmigrantes. Estudio desde un enfoque educativo intercultural" (20.19-2021) y al proyecto REDES N 170039 del Programa de Cooperación Internacional CONICYT.
} 


\section{EDUCACIÓN Y DIVERSIDAD CULTURAL}

Cuando se habla de educación puede llevar a una serie de supuestos muy diversos producto de la polisemia del propio concepto. Esta misma diversidad ha significado una barrera en el avance de los estudios de educación intercultural en las instituciones educativas formales y no formales; que no han logrado iluminar un modelo, paradigma o enfoque de educación que integre una dimensión pedagógica y cultural.

Este planteamiento puede ser evidenciado, por ejemplo, cuando se hace referencia a los grupos culturales presentes en las escuelas, donde la cultura es vista desde una concepción estática y las formas de expresión de estas culturas constituyen un conocimiento embazado que puede ser transmitido a través de eventos puramente folklorizados.

En el artículo, asumimos dos características de la educación, la primera, su carácter social y la segunda, su carácter pedagógico. En el primer caso, la educación puede ser explicada desde las nuevas configuraciones sociodemográficas, la aparición de nuevas demandas sociales, la coexistencia de culturas, en definitiva, la educación como una cuestión política. En el segundo caso, ha existido un cambio en la pedagogía, especialmente, desde el movimiento de la educación crítica que ha llevado a plantear la educación como un acto de transformación de la realidad, que excede la perspectiva institucional. En este sentido, la educación se enmarca en contextos sociales muchas veces marginales y problemáticos, se define como un sistema abierto y se reformula como una actividad social. Citando a Castillejo (1994, p. 16) "cada humano que nace lo hace en un contexto definido-espacio, tiempo, cultura- y es desde esta instancia desde la que se educa". Esta distinción toma sentido en nuestra argumentación, en la medida que haremos referencia a grupos culturalmente diferentes; a saber, niños/as migrados ${ }^{1}$ internacionales, con quienes los profesores interactúan a diario. Al hablar de culturas diferentes, los límites parecen diluirse por lo cual requiere de un primer intento por conceptualizar qué entenderemos por cultura. Para Casanova (2002) no se trata de un producto estable ni único, sino un concepto dinámico, cambiante en función de la influencia con otras culturas, realidades y formas de vivir; por lo tanto, la diversidad sería, la expresión plural de las diferencias, nunca traducidas como negación, discriminación o exclusión, sino como reconocimiento y aceptación del otro como parte de una misma entidad colectiva que los incluye (Vilar, 2009).

Por otra parte, las personas construyen realidad desde lo que son, en consecuencia, construyen y reconstruyen sus culturas a partir de su patrimonio cultural, constituyéndose en portadores de cultura. Este planteamiento es importante para la investigación educativa, por cuanto existen diversas corrientes que ponen en cuestionamiento los estudios referidos a la enseñanza del patrimonio en la escuela, como un asunto exclusivo del ámbito artístico, y no como un tema educativo. Contrariamente, Cisneros (2016) señala que la labor docente no sólo estaría en los contenidos o saberes de una disciplina en particular sino en facilitar

\footnotetext{
Somos conscientes de la diversidad de términos y sus excepciones jurídicas y administrativas, sin embargo, preferimos emplear el término "migrados" que hace referencia al proceso de desplazamiento y no "migrante o inmigrante" que expresan un calificativo a la persona. En cualquier caso, preferimos emplear el concepto de "hijos de migrantes" antes que "inmigrantes/migrantes" ya que los niños no se han desplazado por iniciativa propia, sino por la decisión de adultos. También, es conveniente usar el término "extranjeros" que es como administrativamente el MINEDUC informa la matrícula escolar. Con esta sucinta ejemplificación queremos evidenciar la dificultad conceptual, así como los escenarios político/ normativos donde se emplean.
} 
las condiciones y los espacios de convivencia que permitan reconocer a los otros en sus diferencias y singularidades con el propósito de instaurar el valor humano del respeto y la construcción de la sociedad a través de sus múltiples culturas.

Desde esta perspectiva, la cultura debería ser empleada para visibilizar las diferencias de quienes se relacionan en contextos de diversidad cultural. En efecto, la cultura permite el intercambio de significados y sentidos en el proceso pedagógico y didáctico donde median las relaciones entre los integrantes de la comunidad educativa. La educación como práctica social se articula en torno a esquemas culturales que son formas de aproximarse al conocimiento en un marco de valores compartidos. Esto implica una dimensión epistemológica y axiológica de la cultura y del patrimonio cultural.

\section{PATRIMONIO MIGRANTE EN LAS ESCUELAS}

Respecto del patrimonio cultural, UNESCO (2013) lo define como la cultura material e inmaterial, que constituye un significado personal y social, además de un valor que se enraíza en la memoria colectiva otorgando un sentido de pertenencia a un grupo social determinado. Desde esta perspectiva, el patrimonio cultural otorga y crea memoria colectiva, identidad y sentido de pertenencia. Esta misma entidad realiza una especificación del patrimonio material e inmaterial señalando:

El patrimonio cultural material integra: a) los monumentos: obras arquitectónicas, de escultura o de pintura monumentales, elementos o estructuras de carácter arqueológico, inscripciones, cavernas y grupos de elementos, que tengan un valor universal excepcional desde el punto de vista de la historia, del arte o de la ciencia; b) los conjuntos: grupos de construcciones, aisladas o reunidas, cuya arquitectura, unidad e integración en el paisaje les dé un valor universal excepcional desde el punto de vista de la historia, del arte o de la ciencia; c) los lugares: obras del hombre u obras conjuntas del hombre y la naturaleza, así como las zonas, incluidos los lugares arqueológicos, que tengan un valor universal excepcional desde el punto de vista histórico, estético, etnológico o antropológico.

Por patrimonio cultural inmaterial se entiende aquellos usos, representaciones, expresiones, conocimientos y técnicas, junto con los instrumentos, objetos, artefactos y espacios culturales que les son inherentes -que las comunidades, los grupos y en algunos casos los individuos reconozcan como parte integrante de su patrimonio cultural-. Se manifiestan en los siguientes ámbitos: a) tradiciones y expresiones orales, incluido el idioma como vehículo del patrimonio cultural inmaterial; b) artes del espectáculo; c) usos sociales, rituales y actos festivos; d) conocimientos y usos relacionados con la naturaleza y el universo; e) técnicas artesanales tradicionales.

De acuerdo con Marín (2018), el patrimonio cultural destaca por su capacidad para generar encuentros entre culturas, debilitando las fronteras y estimulando la inclusión social en los contextos transnacionales que surgen a partir de la migración intrarregional. Por tanto, las oportunidades para la inclusión social que se presentan desde la promoción del patrimonio se pueden extender a toda la ecología social y en particular a las escuelas, que es donde se juega el destino de las comunidades.

Un elemento esencial, que desde nuestra perspectiva, justificaría la educación en patrimonio cultural en escuelas con familias que han migrado, es lo que señala Chan (2017) al indicar que el patrimonio cultural cobra sentido, cuando se involucra en este proceso a 
los propios actores portadores de experiencias y expresiones, por lo que se identifica en primera instancia a las familias, grupos étnicos, miembros de organizaciones, o portadores de expresiones de valor patrimonial, instituciones que contribuyen salvaguardar, y preservar la diversidad cultural. Si pensamos que en las escuelas comparten niños/as de diferentes países, merece la pena que los profesores aprendan a reconocer en ellos que son portadores de cultura y emplear esos saberes como conocimiento pedagógico diversificando el currículum y contrarrestando el predominio de un currículum mono-cultural.

Pensamos que, acceder por ejemplo, desde las escuelas, a la explicación de las pinturas Naïf inspirada en el arte infantil haitiano, a las artesanías realizadas en hojas de plátano decoradas en vèvè que son figuras empleadas en las ceremonias religiosas de este mismo país; o la eshuva que son rezos cantados del pueblo Huachipaeri del Perú con los que se invoca a los espíritus de la naturaleza; constituye un desafío para la educación.

Ante tanta diversidad de perspectivas que en la práctica ofrece el concepto de patrimonio, resulta previsible que su definición dependa del contexto académico, social y político de quien, o quienes, la plantean. Por lo tanto, tomaremos los planteamientos de Kulemeyer (2016) quien los organiza en distintos grupos. Es importante señalar que partimos del supuesto que el concepto de patrimonio tal como lo entendemos en la actualidad, es un concepto moderno, propio de las últimas décadas, no exento de matices ideológicos y también pragmáticos. Esta diversificación actual del significado y usos del patrimonio determinará las intervenciones de los educadores en torno a cuestiones como las características de los distintos tipos de patrimonio, contenido y calidad de las presentaciones y discursos que se desarrollan en torno a ellos.

La visión primigenia del patrimonio es la más tradicional y hace referencia al patrimonio cultural como un conjunto de bienes materiales e inmateriales que han sido heredados de nuestros antepasados y que han de ser transmitidos de generación en generación. La principal crítica a esta conceptualización es que el patrimonio pareciera ser una herencia, cuyo carácter está dado únicamente por la historia excluyendo los bienes producidos en el presente. Esta concepción le otorgaba a la cuestión del patrimonio una categoría universal que podía ser entendida de la misma manera en todo tiempo y lugar.

Una conceptualización monumentalista se genera por razones de época, políticas y disciplinares asociadas con las personas que las conciben, tienen reconocimiento aquí las grandes obras arquitectónicas de valor universal, construcciones o lugares excepcionales desde el punto de vista histórico, estético, etnológico o antropológico. Desde esta óptica, la apreciación que se hace de estos bienes suele corresponderse a la concepción de la cultura nacional, regional e, incluso, global como un conjunto de bienes representativos cuya entidad ha sido formalizada jurídicamente, es lo que también se entiende como cultura oficial.

Las conceptualizaciones sociales y comunitarias del patrimonio avanzan desde bienes a testimonios (materiales e inmateriales) heredados de la historia y que pertenecen a una colectividad para su disfrute y transmisión a las generaciones futuras. Desde esta perspectiva, no solo se incorpora una nueva temporalidad (presente y futuro) sino una nueva condición de "pertenencia" comunitaria con una función social. Como señala el autor, el patrimonio es una reflexión sobre nuestro pasado y presente: ahora bien, el sujeto del patrimonio es la gente (la sociedad) y sus formas de vida significativas (el patrimonio).

Las definiciones inclusivas/abarcativas ponen el acento en la propiedad, indicando que no existe un único grupo que pueda atribuirse la definición del patrimonio puesto que 
se trataría de un bien público. El riesgo de esta comprensión se encuentra en lo límites que toman los gobiernos para inscribir determinados bienes (materiales e inmateriales) como parte del Estado y el uso que se realiza como instrumento de legitimación del poder público, como proveedor de bienes simbólicos. En la práctica educativa está presente en la instalación de la historia como construcción de una nación/frontera justificada formalmente como de su pertenencia.

Conceptualizaciones como estas se distancian de los actuales procesos de integración de las culturas carcaterizadas precisamente por la imposibilidad de estandarizar y generalizar los matices, las diferencias y especificidades culturales y sociales donde cada grupo constituyó los bienes patrimoniales que pretende rescatar.

Un último grupo de conceptualizaciones son de corte funcional y, desde la perspectiva de Kulemeyer, son aquellas que mejor reflejan los desafíos del presente, el patrimonio está dado por aquellos bienes que pueden ser considerados susceptibles de ser seleccionados con el propósito de ser sujeto y objeto de una práctica educativa patrimonial.

A partir de lo expuesto, el problema radica entonces, en que si este patrimonio no es reconocido por un grupo, la escuela puede convertirse en un espacio que obliga a la renuncia del patrimonio y la herencia cultural de los alumnos migrantes. Esto es lo que ocurre cuando las escuelas se basan en una adaptación unilateral como forma de integración, desencadenando procesos de aculturación (Marín, 2018).

El término aculturación ha sido analizado desde diferentes perspectivas a lo largo del tiempo y no siempre ha tenido la misma acepción (Graves, 1967; Redfield, Linton \& Herskovits, 1936). En sus orígenes, el proceso de aculturación era unidireccional ya que sólo se tenía en cuenta a la población inmigrante que era quien sufría cambios durante el periodo de adaptación. Sin embargo, los nuevos modelos apoyan la teoría de que no se trata de un fenómeno unidireccional sino que tanto la sociedad de origen como la receptora son dos dimensiones independientes que influyen una en la otra. Uno de los modelos de aculturación más conocido es el de Berry $(1997,2005)$, en el que se plantea que un individuo o grupo puede adoptar en su proceso de aculturación cuatro estrategias en función de dos dimensiones: mantener la propia cultura y el contacto con la cultura-sociedad receptora. En ese sentido, Berry (1980) identificó cuatro estrategias de aculturación: asimilación, separación, integración y marginación. La estrategia "integración", se da cuando la persona migrante continua con sus valores culturales de origen y a su vez mantiene relaciones con personas de culturas diferentes; "separación" sucede cuando continua con su cultura pero no mantiene relaciones con otros grupos culturales; "asimilación" cuando la cultura propia del migrante no tiene continuidad y se relaciona con grupos de otras culturas, y "marginación" cuando no hay percepción de pertenencia a ninguno de los grupos culturales.

Por otro lado, otros modelos como el Modelo Ampliado de Aculturación Relativa (MAAR) consideran que las actitudes o las estrategias de aculturación no se perciben ni se desarrollan de la misma forma cuando el contacto entre inmigrantes y sociedad de acogida se produce en los ámbitos periféricos de la cultura, como son el ámbito político, laboral y económico, que cuando afecta a los ámbitos más intermedios o centrales de la realidad sociocultural (Navas et al., 2005; Navas, Rojas, García \& Fernández, 2011).

A partir de los modelos expuestos surgen distintas preguntas, siendo las más importante, cómo las escuelas debieran identificar e incorporar el patrimonio de niños/as y familias que han migrado a las escuelas y cómo este reconocimiento favorecería escuelas más inclusivas. 


\section{ESCUELAS MULTICULTURALES: CUESTIONES DE RECONOCIMIENTO, IDENTIDAD E INCLUSIÓN SOCIAL}

La presencia de distintas culturas en un mismo espacio y tiempo no tiene los mismos orígenes en todos los momentos y lugares. Las motivos de tal presencia varían considerablemente, desde las reivindicaciones de las minorías étnicas, la revitalización de culturas después de procesos de descolonización, las migraciones sean por motivos políticos, económicos, sociales o personales, los flujos informativos que transportan los medios de comunicación, el intercambio comercial y cultural. En este sentido, la educación multicultural nace de una reflexión sobre la presencia en las escuelas occidentales de minorías que, además de necesitar un trato adecuado por la "distancia" entre su cultura y la cultura presentada y representada por la escuela occidental, necesitan una atención especial ante el fracaso continuado cuando acceden a esta última (Ayerbe, 2000). Esta es, precisamente, una de las primeras dificultades que presenta dicho concepto, al pensar que las culturas representan un obstáculo para el aprendizaje, perpetuando la idea de la cultura como déficit o hándicap.

Por otra parte, si adoptamos la idea de la cultura desde esta "diferencia" implícitamente se apoya la idea de que, además de diferentes, son desiguales. En efecto, Para García, Pulido y Montes (1997) en la necesidad de reconocer y atender a las culturas minoritarias en la escuela, se afirma de modo no explícito -y a menudo quizá no intencionado- que todas las culturas no son válidas para el desenvolvimiento social, por lo que deben ser sustituidas por las culturas mayoritarias. Pensamos que el problema radica en la falta de reconocimiento de la cultura de origen de estas familias. Cuando no existe un reconocimiento y valoración del otro, se corre el peligro de resistirse a las posibilidades y desafíos de las diferencias culturales, principalmente en la interpretación de los saberes educativos. Al respecto, Quilaqueo y Quintriqueo (2010) señalan que los sujetos individuales, que a la vez son sujeto y objeto de sus relaciones sociales, buscan construir y realizar su identidad personal mediante intercambios que les permiten comprender la vida social, de allí que las instituciones educativas constituyan espacios propicios para la integración de grupos culturalmente diferentes. Asimismo, la identidad supone un reconocimiento y apropiación de la memoria histórica, del pasado. Un período que puede ser reconstruido o reinventado, pero que es conocido y apropiado por todos. El valorar, restaurar, proteger el patrimonio cultural es un indicador claro de la recuperación, reinvención y apropiación de una identidad cultural (Molano, 2007).

Por otra parte, está la falta de reconocimiento que trae consigo la exclusión educativa que lleva implícita una valoración diferencial entre grupos. Esta exclusión adquiere diferentes connotaciones en la escuela, por ejemplo, atención segregada, fracaso escolar, el ausentismo, entre otros. Para Vélaz de Medrano (2002) se trata de un proceso de apartamiento dentro de la misma escuela bloqueando la participación y la integración a las rutinas establecidas. Para Escudero (2005), la exclusión educativa es un proceso acumulativo que lleva a un acceso desigual a ciertos contenidos, experiencias y aprendizajes escolares (esenciales). Para la propuesta, reconocer categorías como la de exclusión e inclusión permite focalizar los análisis en aquellos elementos que gatillan dichos procesos. Uno de los aspectos más documentados dice relación con la legitimación de concepciones o saberes que la escuela define como valiosos, generando con ello una selección y organización del currículo escolar que solo responde a los intereses de un grupo en desmedro de otros. En tal sentido, educar en patrimonio implica reconocer otros saberes culturales y otorgarles valor social 
generando prácticas más inclusivas. En efecto, reconocer la identidad cultural de personas que han migrado no reporta únicamente un beneficio para ellos, sino que implica un aprendizaje para todos quienes conforman la comunidad educativa, especialmente, desde el desarrollo de valores y normas de convivencia.

Forte (2007) señala que el reconocimiento introduce un elemento de dignidad y autoestima en los individuos que comparten una comunidad diferenciada y a menudo minoritaria o marginada. De esta manera, el reconocimiento implicaría además de un beneficio directo para estos grupos, una potenciación del sentimiento de co-pertenencia y de la cohesión social. Visto así, el reconocimiento modelaría la identidad al situarse en la frontera entre lo individual y social. Mascarell (1997) explica estos planteamientos definiendo la identidad como algo que no es estable ni rígido y donde la dimensión temporal adquiere importancia en la medida que avanza desde el pasado, presente y futuro, según las historias de vida, lugares, territorios y contextos de vida. Este autor afirma que las identidades se transforman en el tiempo y dan vida a las identidades múltiples cuando las personas o grupos diversos conviven en un espacio común. En el caso de las escuelas, los niños y niñas tienen la posibilidad de enseñar a otros quiénes son, de dónde han venido y a partir de estos discursos re-significar prácticas culturales de sus países de origen confrontándolas con las prácticas del grupo mayoritario; solo en ese encuentro las identidades se entrecruzan otorgando nuevo significado. Para Trueba (2001) es evidente la necesidad de desplazar la categoría de "identidad" por la de "identidades múltiples" permitiendo que los niños, niñas y sus familias puedan mantener su identidad de origen y adaptarla a los cambios socioculturales de la cultura local.

Como hemos visto, empleamos el término de multiculturalidad únicamente para hacer referencia a la presencia de culturas en un mismo espacio, sin que esto implique necesariamente una interacción. Las limitaciones a dicho enfoque expuestas, nos hacen plantearnos la idea de educar en patrimonio desde un enfoque intercultural crítico entendido como una estrategia, acción y procesos permanentes de relación y negociación entre personas, en condiciones de respeto, legitimidad, simetría, equidad e igualdad (Walsh, 2009). Desde esta óptica, no se trata de ofrecer una respuesta educativa compensatoria para niños/as migrados, sino por el contrario, se trataría de reconocer en niños, jóvenes y adultos a portadores de cultura, aun cuando el patrimonio cultural esté sereno. Existen trabajos previos (Stefoni, 2014) que han estudiado cómo jóvenes migrantes desarrollan actividades culturales en diferentes espacios públicos, ocupando plazas y parques, los cuales en ocasiones son improvisados como sitios de reunión, escenarios, talleres y salas de afinación; logrando establecer un vínculo entre símbolos y ritos reterritorizalizados que trascienden sus fronteras nacionales. Pensamos entonces que trabajar desde planteamientos críticos de la educación implicaría el reconocimiento de valores, normas y costumbres de niños/as migrados, en consecuencia, de manifestaciones del patrimonio cultural (material e inmaterial) susceptibles de ser educados.

\section{PATRIMONIO CULTURAL MIGRADO: PROPUESTAS E IMPLICACIONES PARA LA PRÁCTICA EDUCATIVA}

Tal como hemos señalado, el patrimonio cultural tendría un valor inclusivo que al ser confrontado con propuestas educativas permitiría reconocer la diversidad cultural que 
encierran las aulas de escuelas multiculturales, especialmente, aquellas que acogen a niños/ as que han migrado. Desde esta óptica, programas educativos en patrimonio cultural podrían ser implementados para potenciar el desarrollo de la identidad individual reconociendo el valor de la diversidad, dentro de una identidad colectiva.

Muchas veces el patrimonio cultural se encuentra sereno, sin embargo, esto no quiere decir que no se encuentre presente. Los niños, jóvenes y adultos que han migrado son portadores de cultura y en la medida que se socializa un valor, bien o producto cultural y se hace llegar igualitariamente a toda la escuela sin distinción de ningún tipo, se estarían promoviendo escuelas inclusivas. Desde esta perspectiva, una labor importante del profesorado ha de ser el desarrollo de competencias para identificar patrimonio cultural.

Estas son muchas y de diversa índole, por ejemplo, habilidades para entrar en un dialogo intercultural reconociendo y legitimando a los diferentes interlocutores; habilidades comunicativas para relacionarse con empatía, generando confianza y estableciendo acuerdos, habilidades para proponer a las familias una participación más activa y democrática en las escuelas, entre otras. Asimismo, trabajar sobre sus propias creencias y actitudes, que los lleven a crear "otros" modos de pensar, ser, estar, aprender, enseñar, soñar y vivir que cruzan fronteras (Walsh, 2009).

En cualquier contexto educativo, el profesorado competente es aquél que en su labor docente y, por tanto, con alumnado, familias, compañeros/as es capaz de poner en práctica una serie de conocimientos, destrezas y actitudes, para poder dar solución a las situaciones reales que se le presenta.

En cuanto a las competencias interculturales, existen tres grandes dimensiones, que representan los componentes básicos (Malik, 2002, p. 18): Conciencia de nuestras creencias, valores y prejuicios o sesgos culturales (actitudes); Conocimiento y comprensión de la visión del mundo del alumnado y, en general, de los grupos culturales diversos (conocimientos), y Desarrollo de estrategias y técnicas de intervención apropiadas (habilidades). Así pues, en base a estos componentes, algunas de las competencias básicas exigibles a la intervención educativa, desde un enfoque intercultural (Aguaded, de la Rubia, Castellón, 2013, p. 348) se refieren a: Creencias y actitudes, conocimientos y destrezas del profesorado acerca de la conciencia que tiene de sus propios valores y prejuicios. Creencias y actitudes, conocimientos y destrezas del profesorado acerca de la perspectiva cultural del alumnado. Creencias y actitudes, conocimientos y destrezas del profesorado acerca de las estrategias culturalmente apropiadas.

Además, de estas competencias serían necesarias competencias interculturales que pusieran en relación el éxito de la relación intercultural. En ese sentido, otros estudios indican que las competencias necesarias para una experiencia intercultural positiva son cinco: empatía cultural, iniciativa social, estabilidad emocional, flexibilidad y apertura (Van der Zee \& Van Oudenhoven, 2000).

Más allá de las competencias enunciadas pensamos que el punto de partida debiera estar en la conexión entre educación patrimonial y participación de la comunidad educativa que se interesan por identificar y socializar el patrimonio de quienes han migrado. Esto implicaría un reconocimiento de su valor, al tiempo que se crean vínculos sociales de integración cultural, de autoestima y de rentabilidad social, más allá de los aspectos puramente folklorizados con los que suele vincularse el patrimonio de las familias. 


\section{REFERENCIAS BIBLIOGRÁFICAS}

Aguaded, E. M., De la Rubia Ruiz, P. \& Castellón García, E. (2013). La importancia de la formación del profesorado en competencias interculturales. Profesorado. Revista de Currículum y Formación de Profesorado, 17(1), 339-365.

Ávila Ruiz, R. M. (2005). Reflexiones sobre la enseñanza y el aprendizaje del patrimonio integrado: una experiencia en la formación de maestros. Revista de Investigación en la Escuela, 56, 43-53.

Ayerbe Echeberría, P. (2000). Educar a todos: Una mirada desde la escuela multicultural.

Berry, J. W. (1980). Acculturation as varieties of adaptation. Acculturation: Theory, models and some new findings, 9, 25.

. (1997). Inmigration, acculturation and adaption. Applied Psychology: An International Review, 46(1), 5-68.

(2005). Acculturation: Living successfully in two cultures.International journal of intercultural relations, 29(6), 697-712.

Casanova, M. A. (2002). Manual de evaluación educativa. Madrid: La Muralla.

Castillejo, J. (1994). La educación como fenómeno, proceso y resultado. En Castillejo, J. L., Vásquez, G., Coloma, A. y Sarramona, J. Teoría de la educación, Madrid, Taurus, 15-28.

Cisneros Estupiñán, M. (2016). Cultura, lenguaje y educación en un mundo diverso: mirada sociolingüística. En J. Arboleda. (Ed.), Simposio Internacional Educación, Lengua y Cultura. (pp. 9-18). Nueva York, The City University of New York (CUNY) Baruch College.

Chan, G. (2017). Diagnóstico del patrimonio cultural intangible de costa rica: instrumento para reconocer la diversidad cultural. Intersedes, 18(37), 1-33.

Escudero, J. M. (2005). Realidades y respuestas a la exclusión educativa. Jornadas sobre Exclusión Social. Exclusión Educativa. Murcia: Cajamurcia.

Forte Monge, J. M. (2007). Multiculturalismo, identidad y reconocimiento. Thémata. Revista de Filosofía, 39, 614-618.

García, F., Pulido R. y Montes A. (1997). La Educación Multicultural y el Concepto de Cultura. Revista Iberoamericana de Educación, 13, 223-256.

Graves, T. D. (1967). Psychological acculturation in a tri-ethnic community. South-western Journal of Anthropology, 23, 337-350.

Kulemeyer, J. (2016). El patrimonio cultural como referencia principal para alimentar la controversia entre peruanos y bolivianos en torno a las respectivas identidades nacionales. Revista Nuestro Noa, (8), 63-73.

Malik, B. (2002). Desarrollo de competencias interculturales en orientación. Madrid: Departamento MIDE II, Facultad de Educación, UNED. Recuperado de: http://www.uned.es/centrointer/ Competencias_inrerculturales.pdf

Marín, J. (2018). Educando en la frontera norte de Chile: El patrimonio cultural desafiando la exclusión social. Estudios Fronterizos, 19(38), 1.

Mascarell, F. (1997). Identitat cultural i ciutat. Revista Barcelona Educació, 6-8.

Molano, O. L. (2007). Identidad cultural un concepto que evoluciona. Opera, (7), 69-84.

Navas, M., García, M. C., Sánchez, J., Rojas, A., Pumares, P. \& Fernández, J. S. (2005). Relative Acculturation Extended Model (RAEM): New contributions with regards to the study of acculturation. International Journal of Intercultural Relations, 29, 21-37.

Navas, M., Rojas, A., García, M. C. \& Fernández, J. (2011). Concordancia entre actitudes y percepciones de aculturación de la población autóctona hacia los inmigrantes magrebíes: Relación con las actitudes de prejuicio. Anales de Psicología, 27(1), 186-194.

Quilaqueo, D. \& Quintriqueo, S. (2010). Saberes educativos mapuches: un análisis desde la perspectiva de los kimches. Polis. Revista Latinoamericana, (26), 337-360.

Quintriqueo, S., Torres, H., Sanhueza, S. \& Friz, M. (2017). Competencia comunicativa intercultural: formación de profesores en el contexto poscolonial chileno. Alpha (Osorno), 45, 235-254. https://dx.doi.org/10.4067/S0718-22012017000200235. 
Ramírez, E. De la Rubia, P \& Castellón, E. (2013). The importance of teacher training in intercultural skills. Profesorado, Revista de Currículum y Formación del Profesorado, 17(1), 339-365.

Redfield, R., Linton, R. \& Herskovits, M.J. (1936). Memorandum on the study of acculturation. American Anthropologist, 38, 149-152.

Stefoni, C. (2014). Perspectiva transnacional en los estudios migratorios. Revisión del concepto y nuevos alcances de la investigación. En Poblaciones en movimiento. Etnificación de la ciudad, redes e integración (pp. 41-65). Santiago de Chile: Ediciones Universidad Alberto Hurtado.

Trueba, H. (2001). Múltiples identidades étnicas, raciales y culturales en acción: desde la marginalidad hasta el nuevo capital cultural en la sociedad moderna. En E. Soriano (coord.), Identidad cultural y ciudadanía intercultural (pp. 17-44). Madrid: La Muralla.

UNESCO, Organización de las Naciones Unidas para la educación la ciencia y la cultura (2013). Recuperado de https://ich.unesco.org/es/que-es-el-patrimonio-inmaterial-00003

Van Der Zee, K. I., \& Van Oudenhoven, J. P. (2000). The Multicultural Personality Questionnaire: A multidimensional instrument of multicultural effectiveness. European journal of personality, 14(4), 291-309.

Vélaz de Medrano Ureta, C. (2002). Orientación e intervención psicopedagógica. Conceptos, modelos, programas y evaluación. Colección Persona-EscuelaSociedad. Ediciones ALJIBE. Málaga, España.

Vilar García, M. (2009). La diversidad cultural en educación desde enfoques multi e interculturales: conceptos y realidades. Sociedad y Discurso, (16), 102-118. https://doi.org/10.5278/ojs.. v0i16.864

Walsh, C. E. (2009). Interculturalidad, estado, sociedad: luchas (de) coloniales de nuestra época. Universidad Andina Simón Bolívar. 